\title{
EVALUATION OF CLADOSPORIUM SOIL MOLDS PATHOGENICITY
}

\author{
N. O. Kravchenko, O. B. Kopylova, O. V. Golovach, O. M. Dmytruk
}

Soil fungi play an important role in the nature. According to modern concepts fungi kingdom is one of the most important components of ecosystems and includes about 1.5 million species [1]. Typically, the fungi biomass in arable or meadow soils is as big as bacteria biomass [2].

Fungi are involved in the destruction of organic matter at soil formation as well as in the processes of biogeochemical transformations of elements (N, P, K, S), and so on. In addition, fungi closely interact with plants, forming mycorrhiza and synthesizing biologically active substances.

One of the prominent producers of biologically active substances are considered to be the representatives of Cladosporium Link genus, first described in 1816, numbering 169 species and being the most common among imperfect fungi [3].

Note, that the specified genus is characterized by high diversity and includes endophytic, saprotrophic and phytopathogenic fungi. In addition, the representatives of Cladosporium genus can be agents of animal diseases $[4 ; 5]$.

The representatives of Cladosporium genus are isolated out from virtually all known substrates - soil, fallen leaves, stems of herbaceous and woody plants, and can be concomitant with opportunistic infections caused by other pathogens [6-8]. In addition, Cladosporium herbarum is known as hyperallergenic agent [9].

The search for new strains of fungi of Cladosporium genus, capable of producing biologically active substances and positive impact on the growth and development of plants and their use as potential agents of biological products is a promising area of agricultural microbiology. Given the fact that among Cladosporium genus not only pathogens of plants but also animals occur, promising strains should be safe for humans and warm-blooded animals.

The objective of our work was to study the properties of soil pathogenic fungi of Cladosporium genus that are characterized by the ability to produce phytohormonal substances and can be used as the basis for biological products with growth-stimulating activity.

Materials and methods. The strains of fungi of Cladosporium genus $S$. cladosporioides (Fresen.) G. A. de Vries. species. C. cladosporioides 525 strain was isolated from soil rhizospheric soil of spring wheat, C. cladosporioides 495 - from the roots of white lupine of Lybid variety.the strains were grown on wort agar-agar $3-4^{\circ}$ by Baling, $\mathrm{pH}$ 6.4-7.0 at the temperature of $25-27^{\circ} \mathrm{C}$.

The suspensions of spore-mycelium fungi mixtures were prepared in sterile isotonic sodium chloride solution following two-times washing from metabolites and deposition of cells by centrifugation for $20 \mathrm{~min}$. At 2000 $\mathrm{rev}$./min. Working concentration of fungi cells in suspensions were set using Goriaiev chamber.

Pathogenic properties of strains were determined in acute experiments on the model of white outbred mice. Mature white mice weighing 18-20 g were administered with spore-mycelium fungi mixtures orally through a tube and by intraperitoneal injection. The doses for oral administration contained $1.0 \times 10^{6} ; 1.0 \times 10^{7} ; 1.0 \times 10^{8}$ cells of soil fungi, for intraperitoneal injection $-1.0 \times 10^{7}$ and $1.0 \times 10^{8}$ cells per animal [10]. Laboratory animals had been were previously adapted to the conditions within 14 days. Care and observations had been conducted daily for 20 consecutive days after the administration of test material $[10 ; 11]$.

At the end of the observation period on behavioural and physiological responses of laboratory animals their compulsory slaughter and mortem were performed, followed by microscopic study of smears-prints of internals and seeding of tissue samples (liver, kidney, spleen) for elective nutrient medium.

Housing, feeding, care and all manipulations with laboratory animals were carried out under the European Convention "For the protection of vertebrate animals used for experimental and other scientific purposes" (Strasbourg, 1986) [12] and "General ethical 
principles of experiments on animals" approved at the first National Congress on Bioethics (Kyiv, 2001) [13]. Experiments were conducted in compliance with the principles of humanity set out in the European Community Directive [14].

The virulence of soil fungi strains was established by the level of dose of viable microbial cells, which causes death in $50 \%$ of infected animals $\left(\mathrm{LD}_{50}\right)$. The criterion for avirulence of microorganisms was the absence of death of mice within 20 days and characteristic for infectious diseases changes in the internal organs of animals by the results of post-mortem examination.

The infectivity (invasiveness) of fungi strains was determined by seeding of tissue samples (liver, kidney, spleen) of compulsorily slaughtered animals on wort agar-agar. The lack of typical for investigated soil fungi strains growth pointed to their non-infectivity.

So, the obtained results indicate the avirulence of soil fungi $C$. cladosporioides 495 and $C$. cladosporioides 525 as the average lethal dose for oral and intraperitoneal administration of their suspensions is $\mathrm{LD}_{50 \text { per os }}$ $>1.0 \times 10^{8}$ cells/animal, LD $_{50} \mathrm{i} / \mathrm{p} \quad>1.0 \times 10^{8}$ cells/animal. The mortem of compulsorily slaughtered white mice of all experimental and control groups showed no visible signs of pathological changes in the internal organs of animals after the administration of soil fungi $C$. cladosporioides 495 and C. cladosporioides 525 (table 2).

By the results of microbiological tests of samples of the internal organs of animals of both experimental and control groups the growth of retro-cultures of studied fungi was not established. Thus, we can conclude that soil fungi C. cladosporioides 495 and $C$. cladosporioides 525 in applied concentrations do not penetrate the internal organs of animals, do not breed in them and do not cause changes typical for infection process. The avirulence of above mentioned fungi strains of Cladosporium genus for warm-blooded animals is confirmed by the data of medium lethal doses by both oral and intraperitoneal administration.

So, according to the results, soil fungi $C$. cladosporioides 495 and C. cladosporioides 525 belong to the group of avirulent microorganisms incapable of invasion into internals of investigated warm-blooded animals and can be used as the basis for growth-stimulating biological preparations. 